\title{
Corticosteroid treatment in pulmonary sarcoidosis: do serial lavage lymphocyte counts, serum angiotensin converting enzyme measurements, and gallium-67 scans help management?
}

\author{
M TURNER-WARWICK, W MCALLISTER, R LAWRENCE, A BRITTEN, \\ PL HASLAM \\ From the Cardiothoracic Institute, Brompton Hospital, and the Royal Marsden Hospital, London; and \\ Southampton General Hospital, Southampton
}

ABSTRACT Thirty two patients with persisting pulmonary sarcoidosis fulfilling defined criteria for treatment were managed according to a standard clinical protocol. In this an attempt was made to achieve and maintain maximal radiographic and physiological improvement with individually titrated doses of corticosteroids. Lavage cell counts, serum angiotensin converting enzyme (SACE) determinations, and gallium-67 scans were planned at standard intervals but were not used in management decisions. The study analysed serial measurements in relation to changes in the clinical measurements. Twelve patients' radiographs showed complete clearing, seven cleared partially, and $13 \mathrm{had}$ partial clearing with evidence of fibrosis. There was no predictive value in the initial lavage lymphocyte counts or the SACE or gallium measurements. Notably, in seven patients, substantial radiographic improvement was observed when the initial lavage lymphocyte counts were normal. Higher initial lavage neutrophil counts $(p<0.02)$, higher initial radiographic profusion scores $(p<0.02)$, and lower vital capacity $(p<0.01)$ and carbon monoxide transfer factor $(p<0.05)$ were related to incomplete clearing. A repeat study of the patients when their radiograph had cleared maximally showed that the levels of lavage lymphocytes, SACE, and gallium tended to fall, but frequently remained raised even in the presence of a normal radiograph or vital capacity or both. On the other hand, however, most of the patients with a normal lavage lymphocyte count showed persisting abnormality of the radiograph, lung function measurements, SACE, and gallium scan (or of at least one of these indices). The interrelationships between changes in clinical indices (radiograph, vital capacity, and transfer factor) and in lavage lymphocyte counts, SACE, and gallium scans showed that concordance was fairly poor in each comparison; lavage lymphocytes showed a greater major discordance than did the other pairs of measurements. Symptom free patients with normal or stable radiographic appearances have been followed for many months and have shown no clinical deterioration despite abnormal lavage lymphocyte counts, SACE, and gallium scans. Radiographic relapse; within the criteria defined, was seen in only four patients during the study; this was reflected in the gallium counts in three and in SACE and lavage lymphocyte counts measurements in two.

It is concluded that serial lavage lymphocyte counts, serum angiotensin converting enzyme measurements, and gallium-67 scans are not consistently more sensitive methods by which to monitor patients with sarcoidosis during treatment than are serial measurements of high quality radiographs and results of standard lung function tests. 
Bronchoalveolar lavage cell counts, serum angiotensin converting enzyme measurements and gallium67 scans of the lung have been promoted ${ }^{1-3}$ during recent years as being superior to chest radiography and standard lung function tests in the evaluation of the "activity" of pulmonary sarcoidosis. Validation of the claimed superiority of these methods of assessment has been unsatisfactory because of the lack of an adequate independent measurement of the activity of the disease against which they can be compared.

In some reports ${ }^{4}$ the definition of the activity of sarcoidosis is based on systemic symptoms, which do not necessarily reflect the activity of disease in the lungs. Moreover, such symptoms are frequently absent in chronic sarcoidosis, in which the pulmonary disease may nevertheless be progressive. Recent reports ${ }^{5-7}$ define the activity of pulmonary sarcoidosis in terms of lavage lymphocyte counts or a positive gallium-67 scan, or both-which, of course, prevent their subsequent evaluation as markers of activity. In particular, it is important to know whether normal lavage lymphocyte counts, serum angiotensin converting enzyme concentration, or gallium scans imply that active inflammation in the lungs is no longer present and that any residual radiographic or physiological abnormality represents "burnt out" irreversible scarring of the lung.

The purpose of attempting to assess the activity of the disease in the lung also has to be examined. It is frequently used in different ways: as synonymous with the supposed need for treatment; to titrate the dose of steroids during treatment (that is, as a measure of reactivity to detect relapse if the maintenance dose is reduced too far), or to provide an early and sensitive indicator of reactivation after withdrawal of steroids. A different study design is necessary for each of these three distinct purposes. It may not be valid to assume that the answer to any one of these questions will be the same for the others.

Not all "active" sarcoidosis needs treatment because in many patients the disorder resolves spontaneously and apparently completely. The objective of steroid treatment must therefore be examined. Less controversial is its use to relieve systemic symptoms or breathlessness. More controversially, many physicians use steroids in persisting sarcoidosis in an attempt to prevent the development of irreversible lung changes. These are generally attributable to parenchymal fibrosis. Whether corticosteroid treatment achieves this end is not known, because not all cases of sarcoidosis with persisting radiographic nodular shadows progress to fibrotic distortion of the lung.

The present study was designed to examine the relationships of lymphocyte and neutrophil percentage counts in lung lavage fluid, serum angiotensin converting enzyme concentrations, and gallium-67counts to standard clinical indices before and during $\frac{}{0}$ treatment. Throughout the study the lavage lympho- $\overline{\frac{\omega}{5}}$ cyte counts, SACE values, and gallium counts were $\widehat{\Phi}$ withheld from the clinicians in charge of the patients and were deliberately not used to alter treatment.

\section{Methods}

TREATMENT POLICY

For the purposes of this study the clinical policy used was as follows: new symptom free patients with sar-. coidosis affecting the lung parenchyma were not $\vec{N}$ treated immediately but kept under supervision for 8 some 12-18 months provided that respiratory symp- $\omega$ toms did not develop. If by the end of this time the 을 radiograph had failed to clear spontaneously treat- $\vec{\nabla}$ ment was introduced. Patients were treated earlier if $\mathbb{\Phi}$ there was substantial radiographic or physiological $\mathbb{\Phi}$ deterioration or if respiratory symptoms developed. Eight patients were treated for these reasons within $12 \stackrel{\mathbb{S}}{\simeq}$ months of presentation.

A standard treatment protocol was used, starting with prednisolone $40 \mathrm{mg}$ for four to six weeks. (depending on the severity of symptoms and the ini-O tial radiographic abnormality) and then reduced in a standard way with the aim of reducing treatment to $10 \mathrm{mg}$ on alternate days so long as the radiographic $\frac{\circ}{\mathrm{D}}$ appearances and lung function were maintained at $\varrho$ the point of maximal improvement. Once a minimum $\overrightarrow{\overrightarrow{0}}$ dose had been established it was maintained for one 3 year before withdrawal was attempted. If respiratory symptoms, lung function measurements or the chest radiographic relapsed, the dose of prednisolone was increased. In practice changes occurred more often in $\frac{0}{9}$ the radiographic appearances than in symptoms, lung $\underset{-}{\times}$ volumes or transfer factor for carbon monoxide 3 (TLCO), so that in fact treatment adjustments were based mainly on chest radiographic appearances. $\frac{\text { 일 }}{3}$ This study is concerned with following patients before and during treatment. The follow-up of patients after withdrawal of steroids will be reported separately.

\section{MEASUREMENTS}

Clinical assessments were made before treatment and at follow up after four and 12 weeks, and three monthly for one year, four monthly for two years, $\omega$ and six monthly thereafter. The sequence for assessments was as follows:

D AY 1

Breathlessness was evaluated on a standard four point scale. $^{8}$

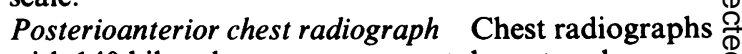
with 140 kilovolt exposures were taken at each assess- $\varrho$ ment. They were read according to a modified 
ILO/UC system ${ }^{9}$ by an individual experienced in its use. The films were read as a batch and without reference to any clinical data or to lavage lymphocyte counts, SACE values, or gallium scans. A profusion score of $0 / 0-3 / 3(0-9)$ was given to each lung, thus defining a $0-18$ total profusion score for both lungs. A count of 3 or less was regarded as indicating a "normal" film. A note was also made of hilar and mediastinal lymph node enlargement and the presence of linear shadows, elevation of the lesser fissure, and hilar vessels suggesting contraction of the upper lobes.

The same films were also read in known order side by side and graded as much better, better, no change, worse, and much worse for each lung separately. There was in fact close agreement between change in the profusion scores and side by side film readings. Concordance for this relationship was $87 \%$ with no major discordant readings.

Standard lung function tests using standard methods were performed at each assessment; they included determination of static and dynamic lung volumes, TLCO, and correction of TLCO for accessible lung volume in 10 seconds (the transfer coefficient-KCo). Progressive exercise tests were also undertaken but the results of these are not included in this report. All results were expressed as percentages of predicted values, the standard values from the tables of Cotes being used. ${ }^{10}$ In the assessment of change in serial studies an arbitary change of $\pm 5 \%$ of the predicted normal value (for example, an increase from $70 \%$ to $75 \%$ of the predicted normal) has been used to define change. The effect of a change of $\pm 10 \%$ was also assessed, but this did not alter correlations significantly.

Serum angiotension converting enzyme assays were carried out by a standard method. ${ }^{11}$ The protocol included measurements at each clinical assessment. The upper limit for our laboratory was 52 $\mathrm{nmol} / \mathrm{ml} / \mathrm{min}$ and a change of 5 units was used as an arbitrary indicator of change in serial assessments.

\section{DAY 2}

Lung lavage methods and the preparation of cell counts have been described previously. ${ }^{12}$ Lung lavage was performed before treatment and repeat lavages were planned so far as possible after one month, three months, and one year, and annually thereafter. For practical reasons unassociated with treatment response, lavage could not be performed on every scheduled occasion. In the study reported here at least two lavages were performed on every patient. The upper limit of normal for percentage cell counts in our laboratory is $11 \%$ for lymphocytes and $4 \%$ for neutrophils. An arbitrary percentage change of 5 has been regarded as "change" in serial studies.

\section{DAYS 4-7}

Gallium-67 scores About 70-80 MBq of gallium-67 citrate was given intravenously 48 hours before gamma camera imaging of the thorax. The images were stored on digital computer and before quantitation areas of interest were outlined to include the lungs and omit the hilar regions and mediastinum. The mean counts for both lungs were expressed as counts/pixel/75 MBq dose/500 seconds. The upper limit for control lungs with this system was 45 counts/pixel/75 MBq dose/500 s. Controls were obtained from patients having gallium scans of other organs who had no evidence of lung disease.

Gallium scans were planned before treatment and after one and three months and one year. Scans were limited to four in any one patient. In practice and for some practical reasons, fewer scans were performed in individual patients. In the study reported here all patients having at least two gallium scan results have been included. The study was approved by the hospital ethics committee and full consent was obtained from patients. A change of 5 units was used as an arbitrary indicator of change.

\section{PATIENTS STUDIED}

Twenty six untreated patients were studied before and during treatment with corticosteroids. A further six patients, who had relapsed while having a small dose of corticosteroids and who were studied before this dose was increased, were also included. They were followed over a mean period of 19.8 months (range 3-64 months). As explained above and for practical reasons, not every patient had a complete set of measurements on every occasion, and the totals vary with the availability of the appropriate data. The numbers of each type of assessment are shown in table 1.

Table 1 Numbers of measurements performed on individual patients $(n=32)$

\begin{tabular}{|c|c|c|c|c|c|c|c|}
\hline & \multicolumn{6}{|c|}{ No of assessments per patient } & \multirow{2}{*}{$\begin{array}{l}\text { Total No of } \\
\text { assessments }\end{array}$} \\
\hline & 7 & 6 & 5 & 4 & 3 & 2 & \\
\hline $\begin{array}{l}\text { Radiograph } \\
\text { Lung function } \\
\text { Lung lavage } \\
\text { SACE } \\
\text { Gallium scan }\end{array}$ & $\begin{array}{l}1 \\
1 \\
0 \\
0 \\
0\end{array}$ & $\begin{array}{l}4 \\
4 \\
1 \\
0 \\
0\end{array}$ & $\begin{array}{l}6 \\
6 \\
6 \\
4 \\
0\end{array}$ & $\begin{array}{r}10 \\
10 \\
9 \\
16 \\
6\end{array}$ & $\begin{array}{l}11 \\
11 \\
12 \\
11 \\
23\end{array}$ & $\begin{array}{l}0 \\
0 \\
4 \\
1 \\
3\end{array}$ & $\begin{array}{r}134 \\
134 \\
116 \\
119 \\
99\end{array}$ \\
\hline
\end{tabular}

SACE - serum angiotensin converting enzyme concentration. 
Of the 32 patients studied ( 12 of them male), 27 were Caucasian, four were Asian, and one was black. Twenty three $(72 \%)$ were non-smokers, six were exsmokers, and only three were smokers. The mean age at the time of first lavage was $38 \cdot 2$ (SD 10.3) years and the mean duration of known abnormality on the chest radiograph was 3.6 (SD 3.9) years. Histological verification of sarcoidosis was obtained in 28 patients; the Kveim test gave a positive result in a further two. Two patients had a typical radiograph with bilateral hilar nodes and parenchymal shadows but did not have histological verification.

\section{ANALYSIS OF RESULTS}

Data are expressed as mean values with standard errors of the mean in parentheses. Unpaired student's $t$ tests have been used to obtain probability values for comparisons of measurements between groups, as indicated in the figures. All significant differences were confirmed with non-parametric Mann-Whitney tests. Paired $t$ tests were used for within group comparisons. The $\chi^{2}$ test was used for data on groups of patients. Ninety five per cent confidence limits were used in assessment of significance. The probability values of significance are included as a guide, but because of the small numbers these values should be interpreted throughout this paper with caution.

\section{Results}

Lavage cell, SACE, and gallium assessments before treatment for predicting the extent of subsequent radiographic clearing

After treatment the chest radiographs of 12 patients showed complete clearing (four patients initially with minimal treatment) (group I). Seven patients (two initially with minimal treatment) showed partial clearing with a persistent profusion score (group II). Thirteen patients showed partial clearing but had persisting linear shadows with evidence of contraction (group III); 11 of these also had a persistent profusion score. The pretreatment data are summarised in table 2 . The ages of the patients in all three groups were similar. Groups II and III together had a slightly longer $\frac{D}{\sigma}$ duration of known disease than group $I$, a higher $\frac{\bar{\sigma}}{\bar{N}}$ radiographic profusion score $(\mathrm{p}<0.05)$, a lower vital $\widehat{\nabla}$ capacity (VC) $(\mathrm{p}<0.02)$, and TLCo $(\mathrm{p}<0.05)$, and a greater number of neutrophils in the lavage fluid os $(p<0.02)$. The mean SACE concentration and per- $\vec{\circ}$ centage lavage lymphocyte counts were increased in $\overrightarrow{\vec{H}}$ all groups without a statistical difference between $\stackrel{\omega}{\sigma}$ them. Likewise there was no difference in the propor- $\vec{x}$ tion of patients in each group with an initial lymphocyte percentage count of more than $28 \%$. In particular, there were seven patients with normal initial $\vec{N}$ lavage lymphocyte counts who nevertheless showed $€$ substantial radiographic clearing with treatment (the $\omega$

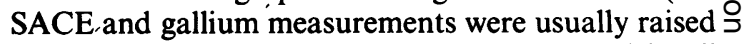
in these patients). Gallium counts were raised in all $\vec{r}$ groups and the mean count was greatest in group III; $\mathbb{\mathbb { D }}$ the data, however, were obtained on an incomplete series of patients and the difference did not reach $\frac{3}{0}$ significance.

Do improvements in serial lavage cell, $S A C E$, and gallium measurements parallel maximum radiographic improvement?

Maximum radiographic clearing was observed over a median of nine (range 1-64) months from the start of treatment (fig 1).

In group I (complete clearing) the mean VC remained $\stackrel{2}{\vec{*}}$ normal (fig 2); slight improvement occurred in TLCO $\frac{3}{3}$ but the mean value remained abnormal (fig 2). The mean lymphocyte percentage count did not fall significantly and remained raised (fig 3 ); $27 \%$ of patients continued to have a count of more than $28 \%$. 음 The lavage neutrophil percentage count remained virtually normal both before and after clearing of the chest radiograph (fig 4). The mean gallium counts (fig 5) and SACE concentrations (incomplete series, fig 6) returned to normal but there was wide individual variation.

Table 2 Pretreatment data

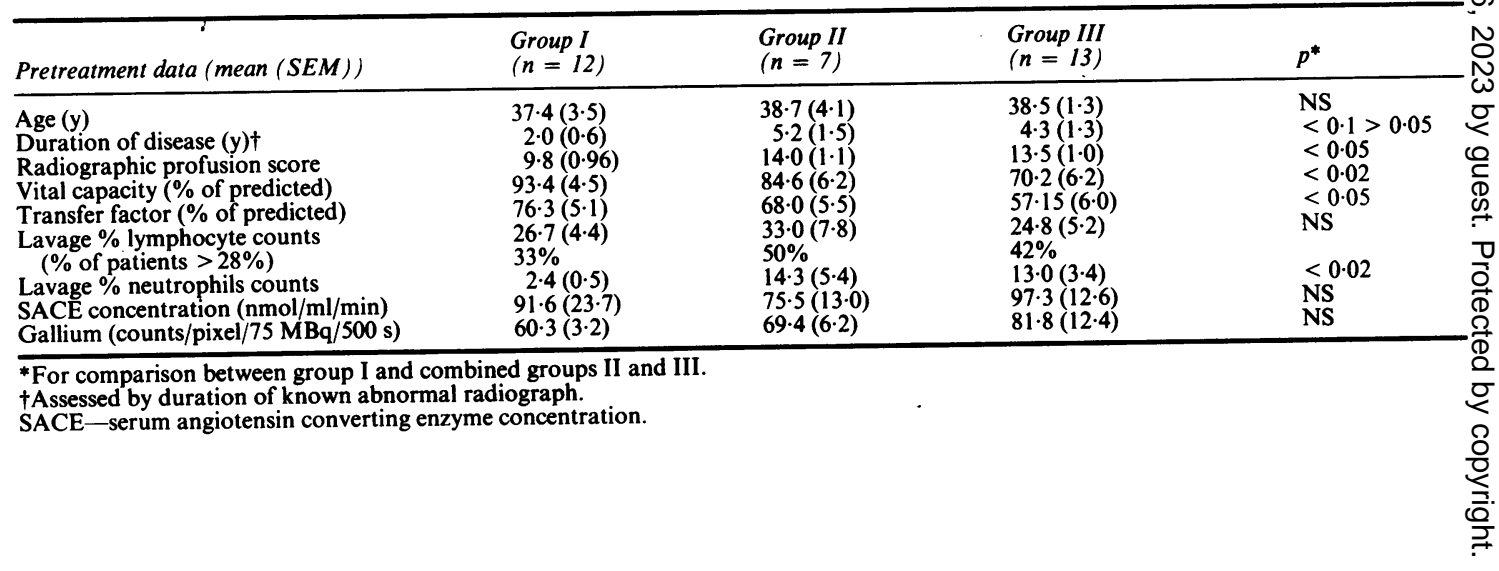




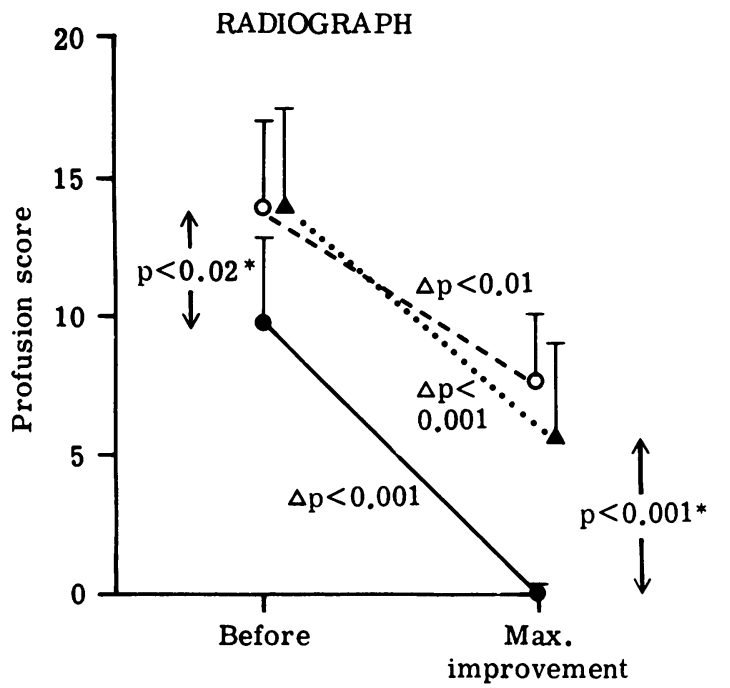

Fig 1 Improvement in radiographic profusion score after treatment in patients with sarcoidosis.

Group I ( - - complete radiographic resolution; group II ( О...... - ) -partial radiographic resolution; group III ( $\mathbf{\Delta}$..... $\mathbf{\Delta})$-partial radiographic resolution with evidence of scarring. * Comparison of group I v group III. $\Delta p-p$ value for comparison (paired t test) between profusion scores before and after treatment.

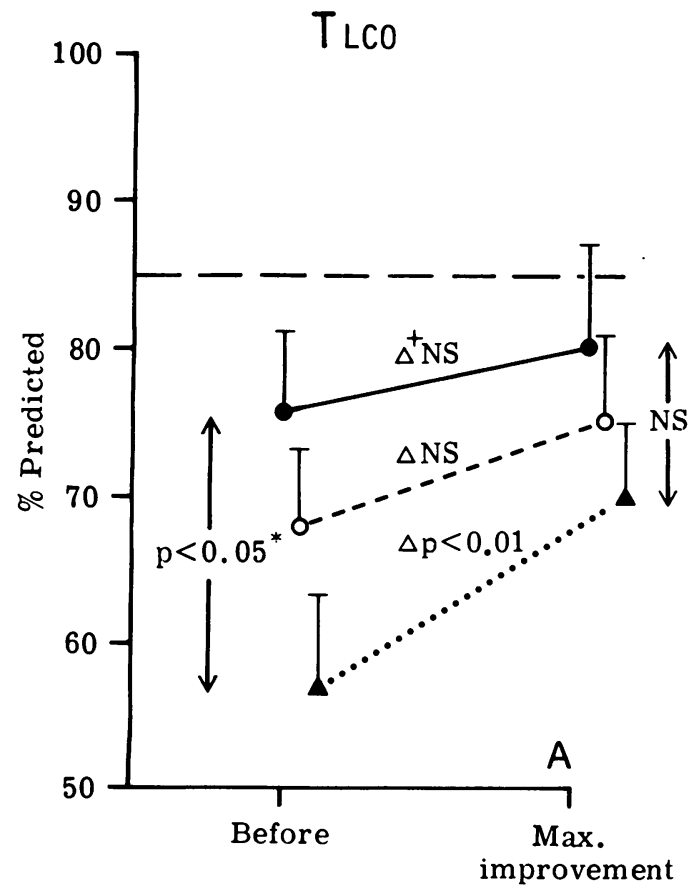

In groups II and III the mean vital capacity returned to normal (group III increasing significantly$\mathrm{p}<0.01$ ). There was improvement of TLCO, which was significant in group III ( $\mathrm{p}<0.01)$, but mean values remained abnormal (fig 2). The lavage lymphocyte count fell significantly $(p<0.001)$ in group II but the change was not significant in group III (fig 3 ). Nevertheless, counts of more than $28 \%$ persisted in three of seven (43\%) and two of $12(17 \%)$ respectively. The neutrophil percentage counts remained raised in both groups and did not change significantly (fig 4). Gallium counts fell, although the small initial numbers prevented statistical comparisons (fig 5). The SACE concentrations fell significantly $(p<0.02$ and 0.01 respectively) to normal (fig 6).

When group I was compared with groups II and III (or group III alone) at the time of maximal radiographic improvement no significant differences in mean values for any measurement could be found. There were, however, a greater number of patients with neutrophil counts under $10 \%$ in groups II and III than in group $I(p=0.05)$. We conclude that, in general, mean lavage cell counts, SACE concentrations, and gallium counts, tend to return towards normal as radiographic improvement occurs.

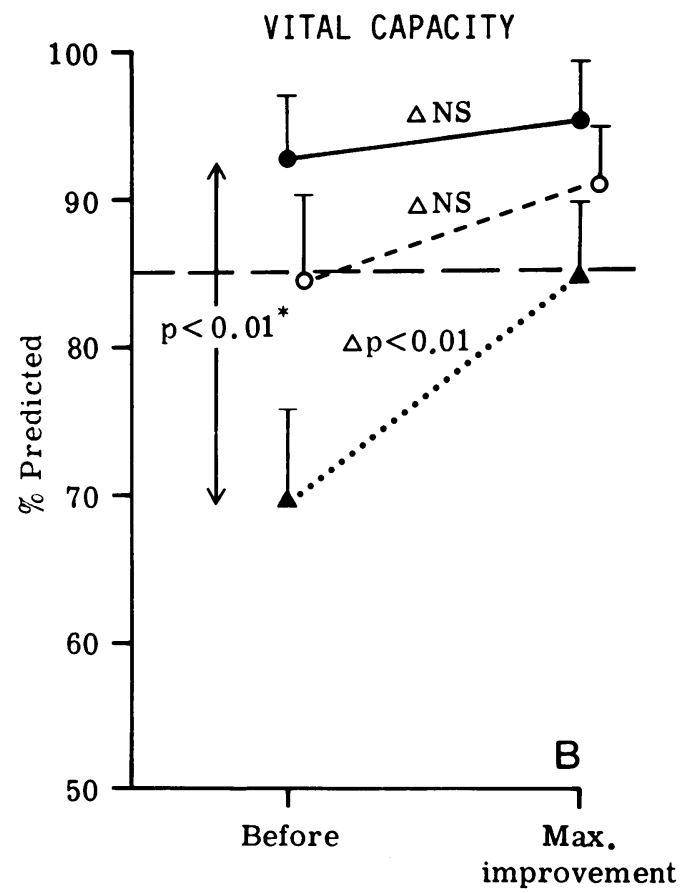

Fig 2 Transfer factor (TLCO) and vital capacity (means and standard errors) in patients with sarcoidosis before treatment and at the time of maximum radiographic resolution. ${ }^{*}$ Comparison of group I v group III. +Indicates statistical comparison before treatment and at the time of maximal radiographic improvement (paired test). Symbols as in figure 1. 


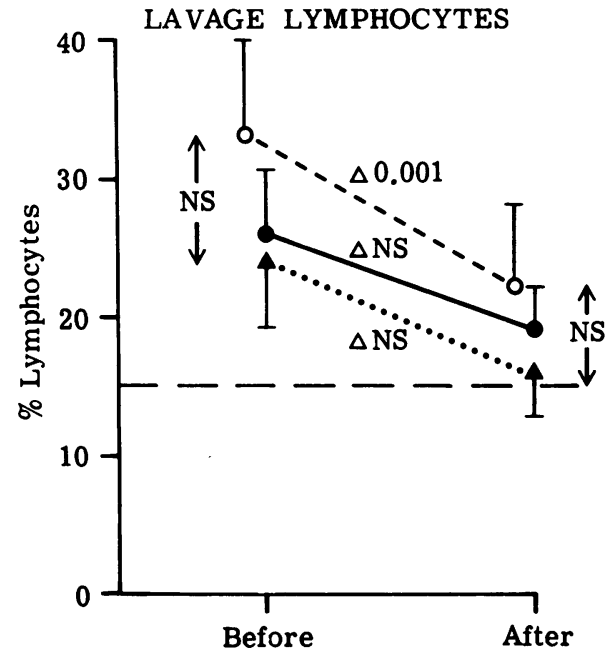

Fig 3 Lavage lymphocyte counts (means and standard errors) in patients with sarcoidosis before treatment and at the time of maximum radiographic resolution. $\Delta-p$ value for comparison (paired t test) between counts before and after treatment.

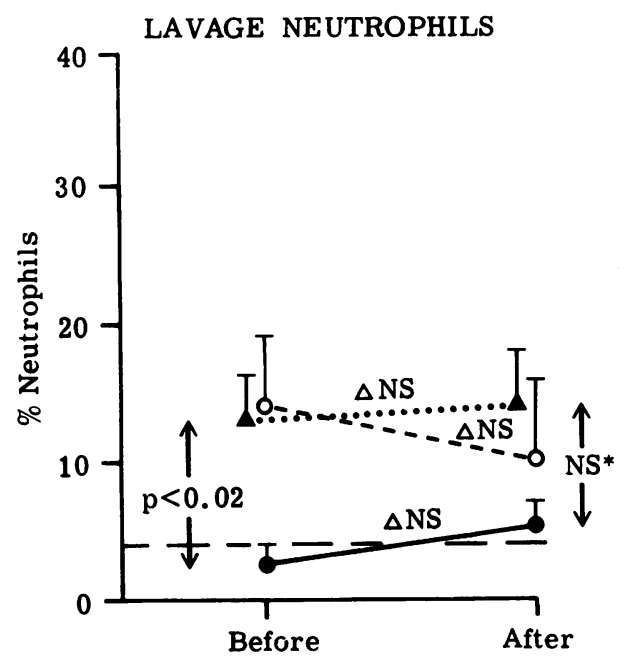

Fig 4 Lavage neutrophil percentage counts (means and standard errors) in patients with sarcoidosis before treatment and at the time of maximum radiographic resolution. Symbols as in figure 1 .

Are normal radiographic appearances and normal lung function accompanied by normal lavage lymphocyte counts, SACE concentrations, and gallium counts? For studying this question data were analysed where all measurements were completed on a given individ- ual patient on the same occasion. During the course of the study some patients had normal radiographic appearances and lavage lymphocyte, SACE, and gal- $\frac{\bar{\omega}}{-}$ lium results on more than one occasion. The radio- $\mathbb{\mathbb { D }}$ graphs from nine patients were scored as normal in $15 \mathrm{~g}$ instances. Lavage lymphocyte, SACE, and gallium

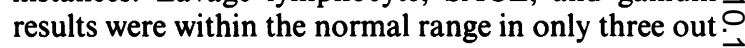
of $15(20 \%)$ instances and were all abnormal in $\vec{\omega}$ another three $(20 \%)$. Lymphocyte counts were $\stackrel{\omega}{\circ}$ abnormal in 10 out of $15(67 \%)$, SACE in seven out $\vec{x}$ of $15(47 \%)$, and gallium scans in six out of $15(40 \%)$.

A similar analysis was undertaken where lung func- $\cdot \vec{\cdot}$ tion measurements were normal and similar results $\vec{v}$ were obtained. For example, the vital capacity was $\mathscr{C}$ within the normal range on 42 occasions; the lavage lymphocyte count was raised in $64 \%$, the SACE con- centration in $38 \%$, and the gallium count in $52 \%$. There were 16 occasions when the radiograph and VCळ were normal; lymphocyte counts were abnormal in $\frac{\Phi}{3}$ $63 \%$, the SACE concentration in $44 \%$, and the gal- 윰
lium scan in $63 \%$.

In summary, the lavage lymphocyte count, $\operatorname{SACE} \vec{\oplus}$ concentration, and gallium scan often remain raised when the radiograph is normal.

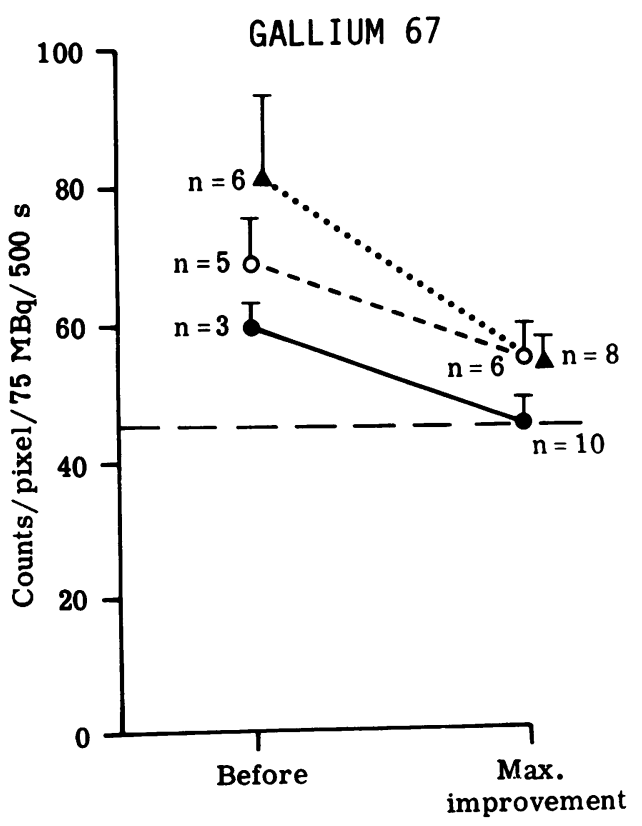

Fig 5 Gallium-67 counts (means and standard errors) in patients with sarcoidosis before treatment and at the time of maximum radiographic resolution. No significant differences $\underset{<}{\sigma}$ were seen. 


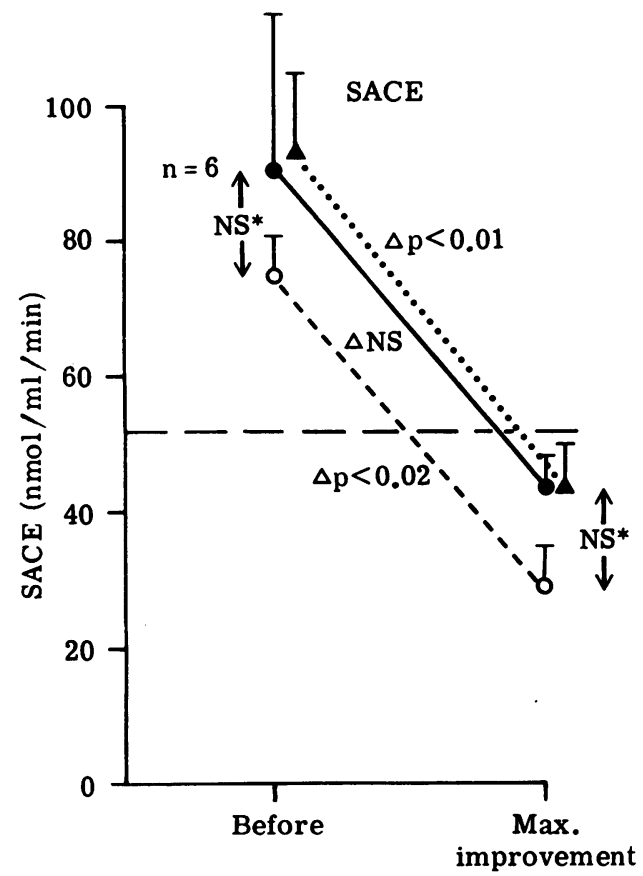

Fig 6 Serum angiotensin converting enzyme (SACE) measurements (means and standard errors) in patients with sarcoidosis before treatment and at the time of maximum radiographic resolution. Incomplete initial data in group I $(n=6)$. Symbols as in figure 1 .

Changes in lavage lymphocyte, SACE, and gallium results and in vital capacity when radiographic appearances were unchanged

There were 23 radiographs from 18 patients in which appearances were unchanged when the patients were followed over a period of at least three (mean 6.2) months and where lavage lymphocyte, SACE, and gallium measurements had been made on the same occasions.

The vital capacity was normal and remained so in 15 out of 23 patients $(65 \%)$. It was initially abnormal in eight ( $34 \%)$ but improved in four. No deterioration in VC was observed in this group. Thus by radiographic and VC criteria the patients were clinically stable (or slightly improving). Nevertheless, many inconsistencies were found when lavage lymphocyte counts, SACE, and gallium scans were considered (table 3). Lymphocyte counts increased in three out of 18 patients $(17 \%)$, SACE values in three out of 23 $(13 \%)$, and gallium counts in seven out of $20(35 \%)$.

Complete data on lavage lymphocytes, SACE, and gallium scans were available in 18 patients at the time of a follow up radiograph with an unchanged score (that is, stable). Completely normal values for all three were found in only three patients $(17 \%)$.

To summarise, the lavage lymphocyte, SACE, sometimes deteriorated when the radiographic appearances and vital capacity remained stable even when followed over several months.

\section{Are normal lavage lymphocyte percentage counts} paralleled by normal values for other measurements?

Before we can conclude that lavage lymphocyte counts are more sensitive measurements of the disease activity than the chest radiograph, changes in the latter must be examined at times when lavage lymphocyte counts are normal.

There were 31 sets of data where the lymphocyte count was less than $12 \%$. The radiographic appearances, SACE concentration and gallium count were also consistently negative in only six $(19 \%)$ of these. The SACE concentration remained raised in eight out of 31 cases $(26 \%)$ and the gallium count in 14 out of $31(45 \%)$. The radiographic appearances were abnor$\mathrm{mal}$ in $22(71 \%), \mathrm{VC}$ in $13(42 \%)$, and TLCo in 22 $(71 \%)$.

An abnormal radiograph might, however, represent irreversible inactive "burnt out" disease. To find out whether this was so, an analysis was made on all cases where the lavage lymphocyte percentage count was normal and where subsequent radiographic follow up data were available for assessing continued progress. The lymphocyte counts were normal in 38 instances. The radiographs taken at the same times

Table 3 Comparison of changes in lavage lymphocyte, serum angiotensin converting enzyme (SACE), and gallium measurements when serial radiographs showed no change (that is, serial films showing same profusion score)

\begin{tabular}{|c|c|c|c|c|c|}
\hline & \multicolumn{2}{|c|}{ Measurement initially normal } & \multicolumn{3}{|c|}{ Measurement initially abnormal } \\
\hline & Remaining normal & Becoming abnormal & $\begin{array}{l}\text { Remaining abnormal } \\
\text { but unchanged }\end{array}$ & Improving & Deteriorating \\
\hline $\begin{array}{l}\text { Lavage lymphocyte } \\
\text { counts }(n=18)\end{array}$ & $7(39 \%)$ & $1(6 \%)$ & $1(6 \%)$ & \multirow{4}{*}{$\begin{array}{l}7(39 \%) \\
(4 \text { to normal) } \\
7(30 \%) \\
\text { (6 to normal) } \\
5(25 \%) \\
(3 \text { to normal) } \\
4(17 \%)\end{array}$} & \multirow{2}{*}{$\begin{array}{l}2(11 \%) \\
(2 \text { remaining }>28 \% \\
1(4 \%)\end{array}$} \\
\hline $\operatorname{SACE}(n=23)$ & $13(57 \%)$ & $2(9 \%)$ & $0(0 \%)$ & & \\
\hline Gallium $(n=20)$ & $1(5 \%)$ & $4(20 \%)$ & $7(35 \%)$ & & $3(15 \%)$ \\
\hline Vital capacity $(n=23)$ & $15(65 \%)$ & $0(0 \%)$ & $4(17 \%)$ & & $0(0 \%)$ \\
\hline
\end{tabular}


Table 4 Percentage concordance and major discordance between change observed between pairs of measurements. 46 comparisons on 28 patients (complete measurements on both occasions)

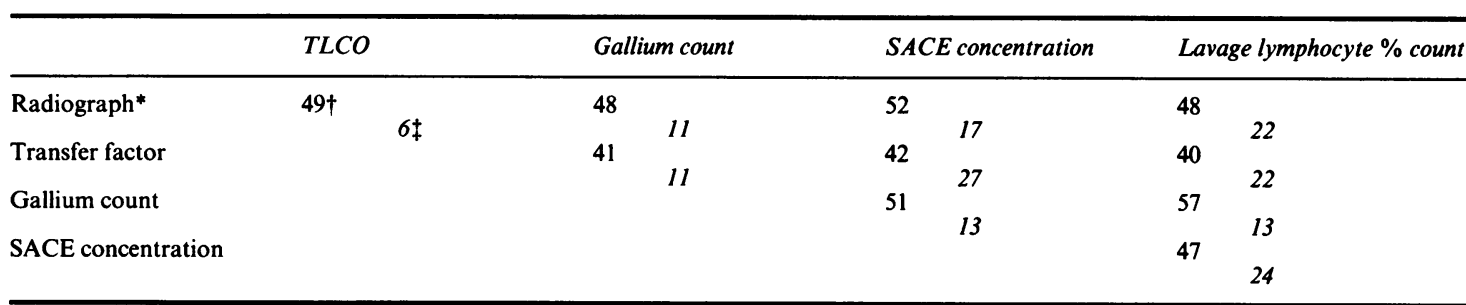

* Radiographic change on the basis of paired film analysis.

$\dagger \%$ concordance - better, no change; worse: see under "Methods" for definitions.

$\ddagger \%$ major discordance (better $v$ worse): italics throughout table.

were scored as abnormal in $33(87 \%)$. Subsequent radiographic improvement during treatment, however, was seen in 21 of the 33 films $(64 \%)$. These results show that a normal lavage lymphocyte count is found frequently in patients where improvement in the radiograph is still possible.

Simultaneous normal values for lavage lymphocyte percentage counts, SACE concentration, and gallium counts were found on 12 occasions. The chest radiograph, however, remained abnormal in seven (58\%), TLCo in eight $(67 \%)$, and VC in three $(25 \%)$. The radiographic appearances subsequently improved in three and TLCO in three. Thus clinical improvement could still occur in some cases when all the "new" methods of assessment might be interpreted as indicating no disease activity.

\section{Lavage lymphocyte, $S A C E$, and gallium}

measurements as predictors of radiographic relapse

There were only four patients in this series who showed a radiographic relapse (increasing score of $\geqslant 3$ ) after an initial improvement. The gallium counts became abnormally high, reflecting the radiographic relapse, in three patients; the SACE concentration in two; and the lavage lymphocyte percentage counts in two. Thus none of these indices detected all the patients showing obvious radiographic relapse.

\section{Concordance between individual lavage lymphocytes, $S A C E$, and gallium scans and clinical measurements during treatment}

In the instances when pairs of complete data were available from an individual patient, analyses of these paired data were performed. We used the arbitrary definition of change by at least 5 "units" for lavage lymphocyte percentage count, SACE concentration, gallium count, and lung function indices, and by at least 3 for the chest radiograph score. Further analysis based on changes of 10 units or more reduced but never eliminated major discordance and did not improve concordance.

Concordance between types of measurements is used to imply agreement regarding improvement, no change, or worsening. Of greater clinical importance are the observations showing a major discordance (that is, one measurement improving while the other worsens, with "no change" ignored). In all, 46 comparisons (for 28 patients) before or during treatment, or both, were available. The concordances and major discordances are summarised in table 4 . All analyses showed a fairly similar and poor concordance, ranging from $40 \%$ to $57 \%$. Gallium and lavage lymphocyte counts correlated best $(57 \%)$ and TLCO and lavage lymphocyte counts worst $(41 \%)$. On the other hand, lavage lymphocyte counts showed a greater major discordance in relation to the radiographic appearances, TLCO, and SACE concentration than most other comparisons.

Does corticosteroid dosage influence measurements?

Corticosteroids may influence the lymphocyte count, SACE concentration, and gallium count either by influencing disease activity or by direct suppressive effects on the responses to the tests per se, effects that are unrelated to the disease.

Eighteen patients had complete or virtually complete measurements before treatment, while they were having the maximum corticosteroid dose (mean 2.6 months), and after the dosage had been reduced.

When patients had been taking maximum doses for one to two months, SACE concentrations and gallium counts returned to normal in more patients than did the radiograph, lung function, or lavage lymphocyte counts. In this group of patients SACE was initially raised in 15 out of $18(83 \%)$ and fell to normal in all but one during maximal corticosteroid treatment. As the corticosteroid dosage was reduced SACE concentrations increased in eight out of 16 $(50 \%)$ patients (usually remaining within the normal range). This was associated with deterioration of the radiograph in appearances or lung function in only one patient. In three out of $16(19 \%)$ patients SACE values were normal during maximum corticosteroid treatment but fell further despite a reduction of dose. 
Fourteen of $16(88 \%)$ patients had abnormal gallium counts before treatment; the counts fell rapidly so that only five out of 15 scans $(33 \%)$ were abnormal with maximum doses of corticosteroid. No counts improved further during follow up but six out of 14 $(43 \%)$ increased as the dose of corticosteroid was reduced. This was associated with deterioration in radiographic appearance or lung function in only one patient.

Lavage lymphocyte percentage counts were abnormal initially in 15 out of $19(79 \%)$ patients but nine out of $19(47 \%)$ counts remained abnormal with the maximum dose of corticosteroid. Five out of 17 $(29 \%)$, however, improved despite the reducing corticosteroid dose; $8 / 17(47 \%)$ increased. This was associated with deterioration in radiographic appearance and lung function in one patient and deterioration of TLCo in two others.

The proportion of patients with raised lavage neutrophil counts increased during maximum corticosteroid treatment from nine out of $19(47 \%)$ to 11 out of $19(58 \%)$. Improvement occurred, however, in 10 out of $17(59 \%)$ as the dose of corticosteroid was reduced. Neutrophil counts increased later in only two of $17(12 \%)$ patients.

The initial chest radiograph was abnormal in 17 out of $18(94 \%)$ patients, but in 14 out of $18(78 \%)$ it remained abnormal with the maximum dose of corticosteroid. Nevertheless, many of these patients showed later improvement $(9 / 17-53 \%)$ despite reduction of the steroid dose. Only two out of $17(12 \%)$ showed deterioration, confirmed in both by lung function measurements; the gallium measurement was concordant with the radiograph in one and the lavage lymphocyte count in the other.

The vital capacity was initially abnormal in fewer patients $(9 / 19-47 \%)$. It remained abnormal in the majority of these on the maximum dose of corticosteroid $(8 / 19-42 \%)$. Six of $17(35 \%)$ subsequently improved and three of $17(18 \%)$ deteriorated; lymphocytes increased in one of these three but otherwise lavage cell counts, SACE, and gallium scans did not follow changes in VC.

TLCo was initially abnormal in 15 out of $19(79 \%)$ patients and failed to return to normal in any patient during maximum corticosteroid treatment. Later improvement occurred in six out of $17(35 \%)$, and a similar number deteriorated. In three of the latter, lymphocytes increased; there were no instances of concordance with SACE or gallium measurements.

In summary, after one or two months of maximum doses of corticosteroids gallium counts and SACE concentrations returned to normal more rapidly than did lavage lymphocyte counts, radiographic appearances, or lung function. The latter three indices often continued to fall towards normal values more slowly, despite decreasing doses of corticosteroids.

A few patients were studied after a relapse in either the radiographic appearances or the lung function measurements. In general, lavage lymphocyte, SACE, and gallium results did not follow in any consistent manner.

\section{Discussion}

Before we discuss the meaning of the findings in this study the validation of lavage lymphocyte, SACE, and gallium measurements must be considered. The cytospin method for collecting lymphocytes has been criticised because it may lead to lower lymphocyte counts than those obtained from micropore filters. ${ }^{13}$ Counts obtained with our technique have been compared with counts from 1 micron sections prepared for electron microscopy, and they agree closely. Consistently normal or abnormal serial lymphocyte counts in individual patients in the present study further suggest that normality and abnormality are characteristics of the individual, and that counts do not reflect random variation due to the method of cell preparation.

Whether the arbitrary limit of 5 "units" for defining change in these three indices is appropriate is unproved. It has been shown that multiple washes of different lobes from the same patient at the same time may give results that vary more than this. ${ }^{14}$ When a 10 "unit" limit of change was used, however, the concordance between radiographic or physiological change and lavage lymphocyte percentage counts was not improved; although major discordance was reduced, it was never eliminated. Concordance was also studied by comparing normal and abnormal values, but this did not improve the agreement.

There is much controversy about the best way of quantifying gallium scans. The semiquantitative method for estimating the intensity and the proportional area of abnormality ${ }^{1}$ was not satisfactory because it did not take into account the implicit variables in the technique regarding the actual dose given, counting times, and counting areas. For these reasons we developed a technique based on standardised actual counts, similar to that recently described. ${ }^{15}$ This has obvious advantages when serial gallium scans are to be compared.

Complete data over long periods of time requiring repeated, multiple measurements, are difficult to collect. This is particularly the case when routine laboratory services are used. Further, where some tests lead to some albeit minor discomfort and others have a potential radiation hazard, modifications of the protocol have to be made for individual patients, in line with good clinical standards of medical practice. The data reported here are subject to these limitations, but 
there is no evidence that incompleteness of the data has led to any particular bias.

The special feature of the design of the present study is the recording of serial lavage lymphocyte, SACE, and gallium data at the same time as clinical measurements, while only the clinical measurements were used to adjust corticosteroid treatment. We make no claim that the initial treatment protocol or the titration of the dose to maintain maximum radiographic and physiological improvement is the correct form of management, but its use has allowed the lymphocyte, SACE, and gallium measurements to be compared with these independent clinical yardsticks.

The studies comparing initial lavage lymphocyte, SACE, and gallium measurements with subsequent radiographic and physiological change over fairly long periods of time, although limited by small numbers, appear to identify some factors associated with incomplete clearing. These include a more severely abnormal initial radiograph and impaired lung function, and the presence of abnormal numbers of lavage neutrophils. The fact that there is little difference in the substantially raised initial lavage lymphocyte counts, SACE concentrations, and gallium counts whether or not the radiograph clears suggests that the activity of granuloma (if these measurements reflect this) is indistinguishable initially in the different types of case and does not relate to the improvement produced by corticosteroids in the radiographic appearance. This favours the hypothesis that some other process, perhaps reflected by the increased neutrophil percentage count, relates to the development of irreversible changes.

The follow up studies using our treatment regimen have produced some instructive and apparently conflicting data. There is good evidence that the chest radiograph can show complete clearing of a substantial abnormality while the SACE concentration, gallium count, and particularly the lymphocyte lavage count continue to be abnormal. Whether this implies that the dose of prednisolone should have been increased until these three indices return to normal or whether corticosteroids in any acceptable dose will not correct abnormalities of these indices is unknown. While our studies do show a relationship between gallium counts and corticosteroid dosage, this may be due to a direct suppressive effect on the measurement rather than supression of disease activity. This interpretation of the data is supported by the follow up studies, which showed no further improvement in counts as the dose of corticosteroid was reduced, even though there was often improvement in the chest radiographic appearances and in vital capacity. On the other hand, there is substantial evidence that the lavage lymphocyte count may be normal when corticosteroid treatment (in doses used in this study) is followed by substantial further clearing of the radio- $\frac{\overline{\bar{N}}}{\bar{D}}$ graph. In this respect, lavage lymphocytes must be $\stackrel{\varnothing}{\unrhd}$ regarded as a less sensitive index of disease activity. The presence of continued activity with a normal lym- $\vec{a}$ phocyte count was confirmed by abnormal SACE concentrations and gallium counts in about two $\overrightarrow{\vec{\omega}}$ thirds of the cases.

We conclude that different aspects of the pathology

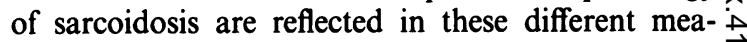
surements, and the changes seen in comparison of serial radiographs may not, in fact be reflected in either the lavage lymphocyte percentage count, the $\underset{\omega}{\mathscr{C}}$ SACE concentration, or the gallium count taken 을 alone.

For clinical purposes, it is not yet known whether it $\bar{\sigma}$ is important to treat patients in such a way as to correct abnormal lavage lymphocyte, SACE, and $\frac{\mathbb{}}{3}$ gallium values completely or even whether this is possible with oral corticosteroids. The radiograph of a symptom free patient, however, appears to be $\vec{\oplus}$ capable of remaining normal for long periods when one or more of these three indices remain abnormal.

Several studies have suggested that changes in the proportions of lymphocyte subtypes (for example, "helper" and "suppressor" cells) correlate better with clinical progress than changes in total lymphocyte $\stackrel{\mathbb{Q}}{\varrho}$ count. $^{16}$ While group differences may be observed, $\vec{\overrightarrow{ }}$ results from individual patients vary considerably, ${ }^{17} 3$ thus lessening the usefulness of these subtypes in monitoring individual patients. Whether other mark-0 ers of lymphocytes will be more useful remains unknown.

If, as has been suggested by some, ${ }^{6}$ abnormal ${ }_{\times}^{\infty}$ lavage lymphocyte values are used to indicate that corticosteroid treatment should be started, the cor $-\frac{3}{6}$ ollary is that patients with normal values will noto receive treatment. The present study shows that sub-

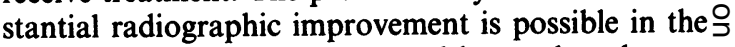
presence of a persistently normal lavage lymphocyte $>$ count. Further, our data do not support the view that only patients with more than $28 \%$ lymphocytes N require treatment if maximal radiographic clearing and symptomatic improvement is the objective.

It may be sensible to ignore lavage lymphocyte $\omega$ counts until their usefulness is proved-that is, untilo they are known to indicate some type of long term, clinically important lung damage. Otherwise, many patients may be exposed to quite unnecessary hazards ${ }^{+}$ from inappropriate large doses of corticosteroids.

There were only four patients showing definite $\frac{O}{\Phi}$

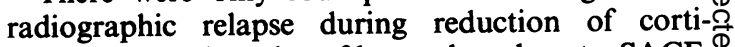
costeroids, so the value of lavage lymphocyte, SACE, $\stackrel{\mathbb{Q}}{2}$ and gallium measurements for detecting early relapse cannot be adequately assessed. Interestingly, gallium $ᄋ$ uptake became abnormal in three of the four patients SACE concentrations became abnormal in only two- 
and this was not therefore a consistent finding, as described by Ainslie and Benatar. ${ }^{18}$ Nevertheless, SACE measurements are simple and facilities are readily available, and a substantial rise in a patient with unchanged clinical features should alert the physician to monitor that patient particularly closely.

This study suggests that, if high quality serial chest radiographs are assessed carefully during treatment of pulmonary sarcoidosis, serial lavage lymphocyte percentage counts, gallium scans, and SACE concentrations do not consistently show a higher sensitivity in indicating change. Such measurements may, however, prove useful in individual cases where simple clinical measurements are discordant, and additional indications of possible activity are needed.

\section{References}

1 Line BR, Hunninghake GW, Keogh BA, Jones AE, Johnston GS, Crystal RG. ${ }^{67}$ Gallium scanning to stage alveolitis of sarcoidosis: correlation with clinical studies, pulmonary function studies and bronchoalveolar lavage. Am Rev Respir Dis 1981;123:440-6.

2 Brambilla C, Chavez L, Brambilla E, et al. Prognostic value of bronchoalveolar lavage and angiotensin converting enzyme in sarcoidosis. In: Chretien J, Marsac J, Saltiel JC, eds. Sarcoidosis and other granulomatous disorders. Paris: Pergammon Press, 1981:419-23.

3 Koontz $\mathrm{CH}$. Lung biopsy in sarcoidosis. Chest 1978; 74:120-1.

4 Klech H, Kohn H, Kummer F, Mostbeck A. Value of different parameters for the assessment of activity in sarcoidosis: $x$-ray, ${ }^{67}$ gallium scanning, serum ACE levels, and blood lymphocyte populations. In: Chretien J, Marsac J, Saltiel JC, eds. Sarcoidosis and other granulomatous disorders. Paris: Pergammon Press, 1981:389-97.

5 Crystal RG, Gadek JE, Ferrans VJ, Fulmer JD, Line BR, Hunninghake GW. Intestitial lung disease: current concepts of pathogenesis staging and therapy. Am J Med 1981;70:542-68.

6 Keogh B, Hunninghake GW, Crystal RG. Therapy decisions in sarcoidosis, prospective use of BAL and
${ }^{67}$ gallium scanning [abstract]. Am Rev Respir Dis 1980;121:151A.

7 Hunninghake GW, Fulmer JD, Young RC, Gadek JE, Crystal RG. Localization of the immune response in sarcoidosis. Am Rev Respir Dis 1979;120:49-57.

8 Medical Research Council Committee on Aetiology of Chronic Bronchitis. Standardized questionnaires on respiratory symptoms. $\mathrm{Br} \mathrm{Med} J$ 1960;ii:1665.

9 International Labour Office. Guidelines for the use of ILO. International classification of radiographs of pneumaconiosis. International Labour Office. Geneva: ILO, 1980. (Occupational Safety Health Series, No 22.)

10 Cotes JE. Lung function: principles and application in medicine. 4th ed. Oxford: Blackwell Scientific Publications, 1979.

11 Friedland J, Silverstein E. A sensitive fluorometric assay for serum angiotensin-converting enzyme. Am J Clin Pathol 1976;66:416.

12 Haslam PL, Turton CWG, Lukoszek A, et al. Bronchoalveolar lavage fluid cell counts in cryptogenic fibrosing alveolitis and their relation to therapy. Thorax 1980;35:328-39.

13 Saltini C, Hance A, Ferrans V, Basset F, Bitterman P, Crystal R. Accurate quantification of cells recovered by bronchoalveolar lavage. Am Rev Respir Dis 1984;130:650-8.

14 Perrin-Fayolle M, Gindre D, Azzard D, et al. The long term evolution of pulmonary sarcoidosis. (Apparent alveolitis compared by lung radiography, gallium lung scans and dual bronchoalveolar lavage analysis.) $R e v$ Pneumol Clin 1985;41:31-7.

15 Alberts C, van der Schoot JB, Groen AS. ${ }^{67}$ Gallium scintigraphy as an index of disease activity in sarcoidosis. Eur J Nuc Med 1981;6:205-12.

16 Ceuppens JL, Lacquet LM, Marien G, Demedts M, van den Eeckhout A, Stevens E. Am Rev Respir Dis 1984;129:563-8.

17 Hughes DA, Haslam PL, Townsend PJ, TurnerWarwick M. Lymphocyte subtypes. Blood and bronchoalveolar lavage $T$ subsets in sarcoidosis and extrinsic allergic alveolitis. Thorax 1984;39:708-9.

18 Ainslie GM, Benatar SR. Serum Angiotensin converting enzyme in sarcoidosis: sensitivity and specificity in diagnosis: correlation with disease activity, duration, extrathoracic involvement, radiographic type and therapy. $Q J$ Med 1985;55:253-70. 